\title{
Métodos de medición de la presión arterial
} Blood pressure measurement methods

Luis Diego Solano Vega', Stephanie Veronica Yagua Velasquez².

1 y 2 Médico general, trabajador independiente.

Contactos: syagua@me.com drdiegosolano@gmail.com

\section{RESUMEN}

La hipertensión arterial es una de las enfermedades más prevalentes a nivel mundial, por lo cual es una de los padecimientos con más estudios realizados para su conocimiento en la epidemiología, fisiopatología y sus riesgos asociados. Es fundamental que todo personal que trabaje en el ámbito de la salud tenga conocimiento básico de esta patología. En la actualidad hay cuatro maneras de medir la presión arterial (PA), dos de ellas es durante la consulta médica y las otras dos no se asocian en el momento de la visita médica. Cada una ellas tienen sus beneficios y sus desventajas, incluso se pueden complementar, lo más importante es saber cuándo utilizar un método u otro dependiendo de la situación y el paciente que estemos valorando.

Palabras Clave: Hipertensión arterial, Medición de presión arterial, Medición automática, Monitorización ambulatoria, Medición domiciliar.

\section{ABSTRACT}

Arterial hypertension is one of the most prevalent diseases worldwide, which is why it is one of the conditions with the most studies carried out to understand its epi-

\section{Cómo citar:}

Solano Vega, L. D., \&

Yagua Velasquez, S.

$\checkmark$. Métodos de medición de la presión arterial. Revista Ciencia Y Salud, 5(3), Pág. 18-22. https://doi. org/10.34192/cienciaysalud.v5i3.279 demiology, pathophysiology and associated risks. It is essential that all personnel working in the health field have basic knowledge of this pathology. Currently there are four ways to measure blood pressure, two of them are during the medical consultation and the other two are not associated at the time of the medical visit. Each of them has its benefits and disadvantages, they can even complement each other, the most important thing is to know when to use one method or another depending on the situation and the patient we are assessing.

Keywords: Arterial hypertension, Blood pressure measurement, Unattended office blood pressure measurement, Home Blood pressure monitoring, Ambulatory blood pressure monitoring.

Recibido: 12/mar/2021

Aceptado: 24/may/2021

Publicado: 22/jun/2021 


\section{CIENCIA\&SALUD}

\section{INTRODUCCIÓN}

La hipertensión arterial (HTA) continúa siendo la mayor causa evitable de enfermedad cardiovascular y de mortalidad por cualquier causa en todo el mundo (1). Se estima que en el mundo hay 1130 millones de personas con hipertensión y la carga global de hipertensión está aumentando debido al incremento de la obesidad y al envejecimiento de la población y se calcula que hacia el 2025 afectará a 1.500 millones de personas, un tercio de la población mundial (2). La prevalencia de la HTA se está acrecentando con más rapidez en los países en desarrollo, donde el diagnóstico tardío y un control inadecuado contribuyen a la epidemia de enfermedades cardiovasculares (2).

El método auscultatorio, realizado por un observador entrenado con un esfigmomanómetro de mercurio, fue el método de elección principal por mucho tiempo en la toma de la PA en consulta, pero ha ido en desuso por su contaminación, lo que hace necesario la utilización de otras alternativas más modernas como la monitorización ambulatoria (1). En la actualidad, los esfigmomanómetros de tipo aneroide y los oscilómetro son más sencillos, baratos y útiles para el diagnóstico de la HTA, aunque estos métodos necesitan ser calibrados periódicamente y validados acorde con protocolos estandarizados (3).

Hay diferentes tipos de pacientes que pueden presentar HTA que se pueden dividir en cuatro clases: La primera es la HTA de bata blanca, la cual se da cuando la PA está aumentada durante la consulta y normal fuera de ella. La segunda es la HTA enmascarada, que se refiere a los pacientes con una PA normal en la consulta y elevada fuera de ella. La tercera es la normotensión verdadera que ocurre cuando la PA medida en consulta y fuera de ella es normal, mientras que la cuarta es la HTA persistente y se emplea cuando en ambas mediciones son anormales (3).

De modo que es de suma importancia en cualquier paciente determinar correctamente sus cifras de tensión arterial, para evaluar los riesgos relacionados. Por eso, conocer cuál de las cuatro técnicas de medición es la correcta y cuándo aplicarla constituye un aspecto de relevancia para el médico tratante, ya que es fundamental obtener la cifra de tensión arterial más precisa y real del paciente.

\section{MÉTODOS Y MATERIALES}

Se realizó una búsqueda de artículos científicos en la base de datos de PubMed y SciELO, además de artículos de la Sociedad Europea de Cardiología (SEC) y de la Asociación Americana del Corazón (AHA) con las palabras clave: "medición de la presión arterial", "hipertensión arterial”, "medición automática y ambulatoria". Se tomó en cuenta únicamente artículos en idioma inglés y español con fechas de publicación desde el año 2009 en adelante.

\section{Métodos de medición de la PA}

En la actualidad hay cuatro maneras de medir la PA, dos suceden durante la consulta médica y dos fuera de ella. Las que suceden durante la consulta son la medición de la PA realizada por el personal médico y la no presenciada. Las otras dos suceden fuera de la consulta o fuera de un ambiente hospitalario: la monitorización en domicilio (AMPA) y la monitorización ambulatoria de la PA (MAPA).

\section{Medición convencional en consulta}

La medición auscultatoria de la PA por personal médico con los esfigmomanómetros semi-automáticos u oscilómetros son el método preferido para la obtención de la PA, estos instrumentos deben estar validados según protocolos estandarizados (4). Por eso es fundamental abordar cómo se obtiene una buena medición de este método.

Para obtener una buena medición, los pacientes deben permanecer sentados cómodamente en un lugar tranquilo durante cinco minutos antes de comenzar la medición de la PA, el manguito de presión debe adaptarse al perímetro del brazo, debe evitarse el consumo de tabaco y la cafeína en los 30 minutos previos (7). Hay que colocar el puño al nivel del corazón, con la espalda y el brazo apoyados para evitar incremen- 


\section{CIENCIA\&SALUD}

tos de la PA dependientes de la contracción muscular y el ejercicio isométrico (14). En la primera consulta, hay que medir la PA a todos los pacientes tras 1 y 3 minutos de bipedestación desde sentado para excluir la hipotensión ortostática, hay que considerar realizarlo en otras consultas en personas diabéticas, personas mayores o con otras enfermedades en la que la hipotensión ortostática es frecuente (5).

Si se usa el método auscultatorio, se debe colocar la membrana del estetoscopio sobre el latido del pulso braquial haciendo una ligera presión, el manguito se infla rápidamente palpando la arteria braquial o radial, hasta $30 \mathrm{mmHg}$ superior a la desaparición del pulso y se debe desinflar despacio menor a $3 \mathrm{mmHg} / \mathrm{seg}$, se utilizan los ruidos de Korotkoff de fase I y $\vee$ para identificar la PA sistólica y diastólica respectivamente (15).

Hay que medir la PA sistólica y diastólica en ambos brazos en la primera consulta para detectar posibles diferencias. Tome como referencia el brazo con el valor más alto. Se deben registrar tres mediciones separadas 1-2 minutos y solo deben repetirse cuando entre las primeras 2 mediciones haya una diferencia mayor a $10 \mathrm{mmHg}$ en la PA sistólica (14). La PA es el promedio de las últimas 2 mediciones. Una diferencia constante y significativa mayor a $15 \mathrm{mmHg}$ de la PA sistólica se asocia con un incremento de riesgo cardiovascular (6), probablemente debido a una patología ateromatosa vascular.

En la práctica, las mediciones en la consulta convencional son imprecisas con frecuencia debido a dos factores significativos: el número bajo de mediciones y la reacción de "bata blanca" (7).

Según la Sociedad Española de Cardiología, se diagnostica HTA cuando la toma de PA con este método se da en múltiples ocasiones y en distintas visitas da mayor a 140/90 mmHg, exceptuando en los casos de HTA grave, como en una HTA grado 3, que es una PA mayor a 180/110 mmHg (7). La definición de la Caja Costarricense del Seguro Social de la hipertensión arterial en adultos es una persona de 18 años o más con presión arterial sistólica (PAS) mayor o igual a $140 \mathrm{mmHg}$ y/o presión arterial diastólica (PAD) mayor o igual a $90 \mathrm{mmHg}$. Para diagnosticar a una persona como hipertensa se requiere de al menos dos valoraciones en diferentes ocasiones espaciadas entre sí por un tiempo prudencial de al menos un mes (16).

\section{Medición automatizada en consulta}

La medición automática no presenciada en consulta registra múltiples lecturas ya que estos equipos se pueden programar para realizar dos o más tomas en intervalos regulares, mejora la reproductibilidad de la medición y más si el paciente se encuentra en una sala aislada, ya que el efecto de la bata blanca puede reducirse sustancialmente o hasta eliminarse (8).

Con este método, los valores de PA son más bajos que los valores obtenidos con la medición convencional en la consulta y pueden ser similares o incluso hasta más bajos que los valores obtenidos fuera de la consulta con la monitorización ambulatoria de la PA (MAPA) o la automedición de la PA (AMPA) domiciliar (9).

La PAS obtenida de forma automatizada en consulta es $15 / 10 \mathrm{mmHg}$ más baja que la PA en la consulta convencional (10).

\section{Monitorización domiciliar}

Los dispositivos electrónicos son los más aconsejables para utilizar en la automedida de la PA, los aparatos pueden ser de brazo, muñeca o dedo, aunque el más aconsejable es el de brazo, ya que mide la PA en la arteria braquial (14). Los que miden la PA en el dedo están desaconsejados por imprecisiones en sus mediciones. Es necesario comprobar el aparato de cada paciente en la consulta para comprobar que le ajuste bien al brazo y que se ha empleado el manguito de un tamaño apropiado, el ancho del brazalete debe ser el $66 \%$ de la distancia entre la axila y el espacio antecubital y su porción inflable debe cubrir al menos el $80 \%$ del brazo (17).

La AMPA es la media de todas las lecturas de PA realizadas con un monitor semiautomático validado durante al menos 3 días, y preferiblemente durante 6-7 días consecutivos antes de cada consulta (11), realizar dos 


\section{CIENCIA\&SALUD}

mediciones en la mañana y otras dos en la noche separadas de cada una por 1 a 2 minutos. Para la toma de estas presiones, el paciente tiene que estar en reposo durante 5 minutos, en posición sentado con la espalda apoyada y el brazo apoyado sobre la mesa a la altura del corazón (14).

Los valores del AMPA suelen ser más bajos que los medidos en consulta, se diagnostica HTA cuando la PA media en el domicilio es de 135/85 mmHg o más. Hay evidencia de que la automedición de la PA puede tener un efecto positivo en la adherencia al tratamiento y el control de la PA (12).

\section{Monitorización ambulatoria}

La MAPA es un dispositivo colocado en el consultorio médico, que proporciona la media de las lecturas de PA durante un período determinado, normalmente es de 24 horas mientras el paciente realiza actividades habituales, incluyendo dormir, es también llamado el Holter de la tensión arterial (2).

El dispositivo se suele programar para registrar la PA en intervalos de 15-30 minutos y para que calcule la media de la PA diurna, nocturna y de 24 horas. El 70\% de las lecturas debe ser satisfactorias para que se considere válida la sesión de MAPA (7). Al igual que el AMPA, los valores de la MAPA suelen ser más bajos que la PA medida en la consulta y el umbral diagnóstico para la HTA es mayor o igual a 130/80 mmHg en promedio durante sus 24 horas de medición, o que su PA media diurna sea mayor o igual de 135/85 mmHg o su PA nocturna media sea mayor o igual de $120 / 70 \mathrm{mmHg}$ (13). Todas estas mediciones equivalen a valores en consulta a mayor o igual a 140/90 $\mathrm{mmHg}$ (7).

\section{Beneficios y desventajas}

La mayor ventaja de la MAPA y la AMPA es que permiten diagnosticar la HTA de bata blanca, HTA enmascarada, normo tensión verdadera y la HTA persistente. Una ventaja muy significativa de la AMPA es su bajo costo y su mayor disponibilidad que la MAPA, además que puede realizar mediciones durante varios días (11). Los dispositivos de MAPA realizan lecturas de la PA durante las actividades diarias del paciente y durante las horas de sueño. En términos generales, ambos métodos deben considerarse complementarios y no como única alternativa (7).

\section{CONCLUSIÓN}

La medición de la PA en consulta es uno de los métodos más utilizados por ser útil, sencillo y de gran valor para el diagnóstico de la HTA, además de dar seguimiento en un paciente ya hipertenso. Sin embargo, este método no es el ideal para diagnosticar diferentes tipos de HTA. Para esto, se prefieren otros métodos de medición y que son superiores como la AMPA y la MAPA. Por lo tanto, es importante el conocimiento de estos métodos de medición de la PA y saber cuándo utilizarlos, ya que con ellos se puede dar un diagnóstico más certero a pacientes mal diagnosticados.

\section{Conflicto de interés}

Los autores declaran ningún conflicto de interés. 


\section{CIENCIA\&SALUD}

\section{REFERENCIAS BIBLIOGRÁFICAS}

1. Achievement of treatment goals for primary prevention of cardiovascular disease in clinical practice across Europe: the EURIKA study, J Banegas, E Lopez-Garcia, J Dallongeville, E Guallar, J Halcox, C Borghi, E Masso-Gonzalez, F Jimenez, J Perk, P Steg, G De Backer, F Rodriguez-Artalejo, 2011.

2. Hypertension, N Poulter, D Prabhakaran, M Caulfield, 2015.

3. ESH/ESC Guidelines for the management of arterial hypertension, G Mancia, R Fagard, K Narkiewicz, J Redón, A Zanchetti, M Böhm, T Christiaens, R Cifkova, G De Backer, A Dominiczak, M Galderisi, D Grobbee, T Jaarsma, P Kirchhof, S Kjeldsen, S Laurent, A Manolis, P Nilsson, L Ruilope, R Schmieder, P Sirnes, P Sleight, M Viigimaa, B Waeber, F Zannad, 2013.

4. A universal standard for the validation of blood pressure measuring devices, G Stergiou, B Alpert, S Mieke, R Asmar, N Atkins, S Eckert, G Frick, B Friedman, T GraßI, T Ichikawa, J Ioannidis, P Lacy, R McManus, A Murray, M Myers, P Palatini, G Parati, D Quinn, J Sarkis, A Shennan, T Usuda, J Wang, C Wu, E O’Brien, 2018.

5. Orthostatic Hypotension Is a More Robust Predictor of Cardiovascular Events Than Nighttime Reverse Dipping in Elderly, R Fagard, P De Cort, 2010.

6. Association of a difference in systolic blood pressure between arms with vascular disease and mortality: a systematic review and meta-analysis, C Clark, R Taylor, A Shore, O Ukoumunne, J Campbell, 2012.

7. ESC/ESH Guidelines for the management of arterial hypertension, B Williams, G Mancia, W Spiering, E Rosei, M Azizi, M Burnier, D Clement, A Coca, G Simone, A Dominiczak, T Kahan, F Mahfoud, J Redon, L Ruilope, A Zanchetti, M Kerins, S Kjeldsen, R Kreutz, S Laurent, G H., R McManus, K Narkiewicz, F Ruschitzka, R Schmieder, E Shlyakhto, C Tsioufis, V Aboyans, I Desormais, 2019.

8. A Short History of Automated Office Blood Pressure - 15 Years to SPRINT, M Myers, 2016.

9. Measurement of Blood Pressure in the Office, M Myers, M Godwin, M Dawes, A Kiss, S Tobe, J Kaczorowski, 2010.

10. Conventional versus automated measurement of blood pressure in primary care patients with systolic hypertension: randomised parallel design controlled trial, M Myers, M Godwin, M Dawes, A Kiss, S Tobe, F Grant, J Kaczorowski, 2011.

11. Role of Ambulatory and Home Blood Pressure Monitoring in Clinical Practice, D Shimbo, M Abdalla, L Falzon, R Townsend, P Muntner, 2015.

12. Telemonitoring and self-management in the control of hypertension (TASMINH2): a randomised controlled trial, R McManus, J Mant, E Bray, R Holder, M Jones, S Greenfield, B Kaambwa, M Banting, S Bryan, P Little, B Williams, F Hobbs, 2010.

13. Diagnostic and Predictive Accuracy of Blood Pressure Screening Methods With Consideration of Rescreening Intervals: A Systematic Review for the U.S. Preventive Services Task Force, M Piper, C Evans, B Burda, K Margolis, E O'Connor, E Whitlock, 2015.

14. Global Risk Assessment to Guide Blood Pressure Management in Cardiovascular Disease Prevention. K Karmali, D Lloyd-Jones, 2017.

15. Effect of Cuff Design on Auscultatory and Oscillometric Blood Pressure Measurements.J Ringrose, D McLean, P Ao, F Yousefi, S Sankaralingam, J Millay, R Padwal, 2016.

16. Caja Costarricense de Seguro Social Guías para la Detección, Diagnóstico y Tratamiento de la Hipertensión Arterial III Edición San José, Costa Rica, 2009.

17. Methods of measurements: home and ambulatory blood pressure monitoring.G Parati, L Krakoff, P Verdecchia, 2010. 